\title{
Avaliação nutricional de mulheres no climatério atendidas em ambulatório de nutrição no norte do Rio Grande do Sul, Brasil
}

\author{
Nutritional evaluation of postmenopausal women \\ treated at a nutrition clinic in the north of the State \\ of Rio Grande do Sul, Brazil
}

Janine Martinazzo ${ }^{1}$

Gabriela Pegoraro Zemolin ${ }^{1}$

Roseana Baggio Spinelli ${ }^{1}$

Vivian Polachini Skzypek Zanardo ${ }^{1}$

Giovana Cristina Ceni ${ }^{2}$

${ }^{1}$ Curso de Nutrição,

Universidade Regional

Integrada do Alto Uruguai e

das Missões. Rua

Vasconcelos 1221.

99.660-000 Campinas do

Sul RS Brasil.

janinemartinazzo@

yahoo.com.br

${ }^{2}$ Curso de Nutrição,

Universidade Federal de

Santa Maria.

\begin{abstract}
The menopause is the stage of a woman's life when the transition from the reproductive to the non-reproductive condition occurs. The objective was to conduct a nutritional assessment of 30 postmenopausal women aged between 40 and 65, by compiling data from the first consultation of nutritional records related to food intake, as well as anthropometric and biochemical data. It was observed that the average result found for body mass index was considered high, being characterized as overweight $\left(30.7 \pm 5.9 \mathrm{~kg} / \mathrm{m}^{2}\right)$ and waist circumference revealed a very high risk for developing cardiovascular disease $(98.2 \pm 15.9 \mathrm{~cm})$. Saturated fats were above the recommended level $(8.14 \pm 3.63 \%)$, whereas monounsaturated and polyunsaturated fats were lower resulting in 6.47 $\pm 3.40 \%$ and $5.37 \pm 2.60 \%$ respectively. The average calcium intake was $549.63 \pm 315.87 \mathrm{mg}$, while vitamin D intake was $549.63 \pm 315.87 \mathrm{mcg}$, both of which were considered inadequate. Triglycerides were classified as optimal (134,3 \pm $85,4 \mathrm{mg} / \mathrm{dL})$, total cholesterol was borderline (223.9 $\pm 141.3 \mathrm{md} / \mathrm{dL}$ ), HDL-C adequate ( $54.3 \pm 18.5 \mathrm{mg} /$ $\mathrm{dL})$ and $L D L-C$ desirable $(128.4 \pm 45.1 \mathrm{mg} / \mathrm{dL})$. The results demonstrate that nutritional care is important and can prevent specific comorbidities in this age group, contributing to the quality of life of menopausal women.
\end{abstract}

Key words Women, Menopause, Nutritional assessment
Resumo O climatério é a fase da vida da mulher em que ocorre a transição do período reprodutivo para o não reprodutivo. O objetivo da pesquisa foi realizar a avaliação nutricional de 30 mulheres no climatério com idades entre 40 e 65 anos, compilando os dados da primeira consulta dos prontuários nutricionais referentes ao consumo alimentar, dados antropométricos e bioquímicos. Observou-se que o resultado médio encontrado para o Índice de Massa Corporal foi considerado elevado, caracterizando excesso de peso (30,7 \pm $5,9 \mathrm{~kg} / \mathrm{m}^{2}$ ) e a circunferência da cintura apresentou risco muito alto para desenvolver doenças cardiovasculares $(98,2 \pm 15,9 \mathrm{~cm})$. As gorduras saturadas estiveram acima do preconizado $(8,14 \pm$ $3,63 \%)$, enquanto que as gorduras mono e poliinsaturada estiveram abaixo, resultando em 6,47 $\pm 3,40 \%$ e 5,37 $\pm 2,60 \%$, respectivamente. O consumo médio de cálcio foi de 549,63 $\pm 315,87 m g$, já o de vitamina $D, 549,63 \pm 315,87 \mathrm{mcg}$ e ambos estiveram inadequados. Os triglicerídeos foram classificados como ótimos (134,3 $\pm 85,4 \mathrm{mg} / \mathrm{dL}), o$ colesterol total limitrofe $(223,9 \pm 141,3 m d / d L), o$ $H D L-C$ adequado $(54,3 \pm 18,5 \mathrm{mg} / \mathrm{dL})$ e o $L D L-C$ desejável $(128,4 \pm 45,1 \mathrm{mg} / \mathrm{dL})$. Os resultados demonstram que o cuidado nutricional é importante, podendo prevenir comorbidades específicas desta faixa etária, contribuindo para a qualidade de vida das mulheres climatéricas.

Palavras-chave Mulheres, Climatério, Avaliação nutricional 


\section{Introdução}

O climatério corresponde à fase da vida da mulher em que ocorre a transição do período reprodutivo para o não reprodutivo. Nessa fase, ocorrem alterações endócrinas devido ao declínio da atividade ovariana, às mudanças biológicas em função da diminuição da fertilidade e às mudanças clínicas consequentes das alterações do ciclo menstrual e de uma variedade de sintomas ${ }^{1}$. O período do climatério considera-se que inicie por volta dos 40 anos e termine na senescência, ou seja, em torno dos 65 anos $^{2}$.

A alimentação equilibrada é fundamental para promover a saúde da mulher climatérica, uma vez que o consumo alimentar inadequado constitui-se como um importante fator de risco para o desenvolvimento de doenças altamente prevalentes nessa fase da vida ${ }^{3,4}$.

Entre as principais morbidades que acometem a mulher no climatério inclui-se a osteoporose, doença de grande impacto tanto na saúde pública como socioeconômico, em função de seus altos custos diretos e indiretos. Além disso, com o aumento da expectativa de vida, cresce também o número de idosos e de fraturas osteoporóticas, particularmente em mulheres ${ }^{5}$.

O baixo consumo de cálcio, nessa fase da vida, é um fator preocupante, pois, embora a osteoporose não apareça entre as principais causas de mortalidade, essa doença constitui-se como um problema de saúde pública em muitos países, devido à sua alta prevalência entre as mulheres climatéricas $^{4-6}$.

A vitamina $\mathrm{D}$ é um hormônio essencial na regulação do metabolismo do cálcio, no desenvolvimento e na manutenção de um esqueleto saudável e também na prevenção de osteoporose. Apenas uma pequena quantidade de vitamina D é proveniente da dieta e a pele é o órgão que mais a produz, sendo, portanto, sua maior fonte ${ }^{7}$.

O cálcio dietético adequado é necessário e pode fornecer proteção adicional contra osteoporose, pois, além de ser necessário para a transmissão dos impulsos nervosos, corresponde a $99 \%$ dos ossos e dentes. A vitamina D tem como principal função a manutenção da homeostase do cálcio, intensificando sua absorção ${ }^{8}$.

Segundo Silva ${ }^{9}$, alterações hormonais neste período são responsáveis pelo aumento de peso e da gordura abdominal, e pela alteração do perfil lipídico, estando já bem estabelecida a associação positiva entre estes fatores e a doença cardiovascular.
Hábitos alimentares inadequados com elevado teor de gordura saturada e colesterol, por exemplo, contribuem para inúmeras doenças crônicas não transmissíveis, entre elas, doenças cardiovasculares, obesidade e dislipidemias ${ }^{10}$.

No Brasil, as doenças cardiovasculares são a principal causa de mortalidade e, comparando as taxas ajustadas por idade das principais metrópoles brasileiras com as de alguns países, notase que, na faixa etária de 45 a 64 anos, várias cidades brasileiras, como Rio de Janeiro, Curitiba e Porto Alegre, apresentam valores elevados, sobretudo entre as mulheres.

Dentro deste contexto, o presente estudo teve por objetivo realizar a avaliação nutricional de um grupo de mulheres no climatério atendidas num Ambulatório de Especialidades em Nutrição de uma Universidade do Norte do RS ${ }^{11}$.

\section{Métodos}

Trata-se de uma pesquisa transversal do tipo retrospectivo, de cunho quali e quantitativo. A amostra foi composta por 30 mulheres atendidas num Ambulatório de Especialidades em Nutrição, no segundo semestre de 2011 e primeiro semestre de 2012, que estavam no climatério, com idades entre 40 e 65 anos e que aceitaram participar da pesquisa, assinando o Termo de Consentimento Livre e Esclarecido.

Este estudo teve a aprovação do projeto pelo Comitê de Ética em Pesquisa. Foram compilados dados da primeira consulta dos prontuários nutricionais, portanto não houve influência do tratamento nutricional. Os dados coletados foram referentes ao consumo alimentar, energia e macronutrientes, com ênfase aos diferentes tipos de lipídios, sendo eles colesterol, gordura saturada, gordura monoinsaturada e gordura poli-insaturada, além dos micronutrientes cálcio e vitamina D; dados antropométricos referentes ao índice de massa corporal (IMC) e da circunferência da cintura (CC); e dados bioquímicos de colesterol total, HDL-colesterol, LDL-colesterol e triglicerídeos.

O consumo alimentar foi analisado através do Recordatório 24 horas (R24h), onde foram avaliados, com o auxílio do software de nutrição $\operatorname{dietWin}^{\circledR}$, versão $2008^{12}$, mediante a comparação com os padrões estabelecidos segundo a $\mathrm{Di}$ etary Reference Intakes (DRI 2002 ${ }^{13}$ para macronutrientes e DRI $1997^{14}$ para os micronutrientes cálcio e vitamina D). Para avaliação dos diferen- 
tes tipos de gorduras, entre eles, gordura satura$\mathrm{da}$, gordura monoinsaturada, gordura poli-insaturada e colesterol, a comparação com os valores de referência foi mediante Carvalho ${ }^{15}$.

$\mathrm{O}$ índice de massa corporal (IMC) foi classificado segundo critérios da Organização Mundial da Saúde (1995/1997) para adultos, e, segundo Lipschitz (1994), para idosos. A circunferência da cintura (CC) de acordo com os parâmetros estabelecidos pela OMS de $1998^{16}$.

Os parâmetros bioquímicos foram classificados segundo a III Diretrizes Brasileiras sobre Dislipidemias e Diretriz de prevenção da Aterosclerose do Departamento de Aterosclerose da Sociedade Brasileira de Cardiologia, de $2001^{17}$.

A análise estatística utilizada foi do tipo descritivo, onde tabelas foram elaboradas, além das principais estatísticas amostrais como média e desvio padrão.

\section{Resultados}

O presente estudo teve a participação de $30 \mathrm{mu}-$ lheres, com média de idade de 50,93 $\pm 6,48$ anos. Pouco mais da metade das participantes possuía menos de 50 anos $(53,33 \%)$ e uma minoria $(13,33 \%)$ possuía mais que 60 anos de idade.

$\mathrm{Na}$ Tabela 1 podemos observar os indicadores socioeconômicos das mulheres climatéricas.

A escolaridade que prevaleceu foi o ensino médio completo ( $40 \%$ ) e a renda de 1 a 2 salários $(33,33 \%)$ se igualando a proporção de 2,1 a 4 salários-mínimos mensais. A ocupação mais relatada foi "do lar" $(43,3 \%)$.

Além dos dados socioeconômicos, $16,7 \%$ das mulheres do estudo relataram possuir hipertensão arterial sistêmica (HAS) e dislipidemias, sendo que $53,3 \%$ do total das participantes referiram possuir alguma história de HAS na família.

A análise dos prontuários revelou que $66,7 \%$ das participantes afirmaram já ter realizado algum tipo de dieta, que o laticínio mais consumido é o queijo $(86,7 \%)$ e que pouco mais da metade $(53,3 \%)$ ingere de 1 a 2 litros de líquidos diariamente. Apenas $16,7 \%$ das participantes relataram adicionar sal na comida pronta, utilizando o saleiro à mesa.

Quanto ao consumo de frituras, 60\% das mulheres do estudo referiram consumir apenas 1 vez por semana, seguido de $20 \%$ que consumiam 2 vezes. O número de latas de óleo consumido em um mês foi de 1 a 2 latas de óleo (60\%).
Em relação a bebidas alcoólicas e tabagismo, $26,7 \%$ e $13,3 \%$ do total de mulheres fazem uso destas, respectivamente.

$\mathrm{Na}$ Tabela 2, estão demonstrados os dados antropométricos das mulheres do estudo.

$\mathrm{Na}$ Tabela 3, estão demonstrados os dados referentes ao consumo alimentar das participantes, sendo que o método utilizado foi o Recordatório Alimentar de 24 horas (R24h).

$\mathrm{Na}$ Tabela 4, estão demonstrados os dados bioquímicos das mulheres climatéricas.

Tabela 1. Indicadores socioeconômicos das pacientes em climatério atendidas no Ambulatório de Especialidades em Nutrição

\begin{tabular}{lrr}
\hline \multicolumn{1}{c}{ Indicadores } & N & \multicolumn{1}{c}{$\%$} \\
\hline Sexo & & \\
$\quad$ Feminino & 30 & $100 \%$ \\
Idade & & \\
$\quad \leq 50$ anos & 16 & $53,33 \%$ \\
$\quad 51$ a 60 anos & 10 & $33,33 \%$ \\
$\quad \geq 61$ anos & 04 & $13,33 \%$ \\
Escolaridade & & \\
$\quad$ Fundamental incompleto & 04 & $13,3 \%$ \\
$\quad$ Fundamental completo & 05 & $16,7 \%$ \\
$\quad$ Médio incompleto & 02 & $6,7 \%$ \\
$\quad$ Médio completo & 12 & $40 \%$ \\
$\quad$ Superior completo & 07 & $23,3 \%$ \\
Ocupação & & \\
$\quad$ Do lar & 13 & $43,3 \%$ \\
Professora & 05 & $16,7 \%$ \\
Cozinheira & 03 & $10 \%$ \\
$\quad$ Outras (vendedora, telefonista, servente...) & 09 & $30 \%$ \\
Renda & & \\
$\quad$ Até 1 salário-mínimo mensal & 06 & $20 \%$ \\
De 1,1 a 2 salários-mínimos mensais & 10 & $33,33 \%$ \\
$\quad$ De 2,1 a 4 salários-mínimos mensais & 10 & $33,33 \%$ \\
$\geq 4,1$ salários-mínimos mensais & 04 & $13,33 \%$ \\
& & \\
& &
\end{tabular}

Fonte: $\mathrm{O}$ autor, 2012.

Tabela 2. Dados antropométricos (média e desvio padrão) das pacientes em climatério atendidas no Ambulatório de Especialidades em Nutrição

\begin{tabular}{lc}
\hline \multicolumn{1}{c}{ Dados Antropométricos } & Média \pm DP \\
\hline Peso atual $(\mathrm{Kg})$ & $79,1 \pm 15,7$ \\
Estatura $(\mathrm{cm})$ & $160 \pm 0,1$ \\
Índice de Massa Corporal $\left(\mathrm{Kg} / \mathrm{m}^{2}\right)$ & $30,7 \pm 5,9$ \\
Circunferência da Cintura $(\mathrm{cm})$ & $98,2 \pm 15,9$
\end{tabular}

Fonte: O autor, 2012. 
Tabela 3. Dados do consumo alimentar dos R24h (média e desvio padrão) das pacientes em climatério atendidas no Ambulatório de Especialidades em Nutrição.

\begin{tabular}{lcc}
\hline \multicolumn{1}{c}{ Nutrientes } & Média \pm DP & Valor de referência \\
\hline Kcal & $1597,43 \pm 521,44$ & \\
Carboidratos\% & $55,38 \pm 7,80$ & 45 a $65 \%{ }^{13}$ \\
Carboidratos (g) & $217,42 \pm 74,08$ & - \\
Proteínas (\%) & $16,55 \pm 3,75$ & 10 a $35 \%^{13}$ \\
Proteínas (g) & $65,67 \pm 24,84$ & - \\
Proteínas (g/Kg peso) & $0,86 \pm 0,45$ & $\geq 0,8 \mathrm{~g} / \mathrm{Kg}^{13}$ peso $^{13}$ \\
Lipídeos (\%) & $28,08 \pm 7,10$ & 20 a $35 \% \%^{13}$ \\
Lipídeos (g) & $50,68 \pm 22,50$ & - \\
Colesterol (mg) & $169,50 \pm 90,15$ & $<300 \mathrm{mg}^{15}$ \\
Gordura Saturada (\%) & $8,14 \pm 3,63$ & $7 \%{ }^{15}$ \\
Gordura Saturada (g) & $14,55 \pm 8,64$ & - \\
Gordura Monoinsaturada (\%) & $6,47 \pm 3,40$ & $13 \%{ }^{15}$ \\
Gordura Monoinsaturada (g) & $11,72 \pm 7,76$ & - \\
Gordura Poli-insaturada (\%) & $5,37 \pm 2,60$ & $10 \%{ }^{15}$ \\
Gordura Poli-insaturada (g) & $9,39 \pm 5,65$ & - \\
Cálcio (mg) & $549,63 \pm 315,87$ & $1000 \mathrm{a} 1200 \mathrm{mg}^{14}$ \\
Vitamina D (mcg) & $549,63 \pm 315,87$ & $5 \mathrm{a} 10 \mathrm{mcg}^{14}$
\end{tabular}

Fonte: O autor, 2012.

Tabela 4. Dados bioquímicos (média e desvio padrão) das pacientes em climatério atendidas no Ambulatório de Especialidades em Nutrição

\begin{tabular}{ll}
\hline \multicolumn{1}{c}{ Dados Bioquímicos } & \multicolumn{1}{c}{ Média \pm DP } \\
\hline Triglicerídeos $(\mathrm{mg} / \mathrm{dL})$ & $134,3 \pm 85,4$ \\
Colesterol total $(\mathrm{mg} / \mathrm{dL})$ & $223,9 \pm 141,3$ \\
Colesterol HDL $(\mathrm{mg} / \mathrm{dL})$ & $54,3 \pm 18,5$ \\
Colesterol LDL $(\mathrm{mg} / \mathrm{dL})$ & $128,4 \pm 45,1$
\end{tabular}

Fonte: O autor, 2012.

\section{Discussão}

O climatério representa a transição da vida reprodutiva para a não reprodutiva, que se inicia normalmente por volta dos 40 anos, estendendo-se até os 65 anos. Embora seja considerado um fenômeno natural, pode ser caracterizado por sinais e sintomas que comprometem a qualidade de vida das mulheres ${ }^{10}$.

Em relação à adição de sal na comida pronta, $16,7 \%$ das mulheres afirmaram esta prática, e, segundo o Guia Alimentar para a População Brasileira ${ }^{18}$, o consumo de até $5 \mathrm{~g}$ de sal/dia por pessoa, é a quantidade suficiente para atender as necessidades de iodo. Também recomenda a não utilização de saleiro à mesa, para evitar o acréscimo de sal à comida pronta.
O número de latas de óleo consumido em um mês, em média, foi de 1 a 2 latas de óleo em $60 \%$ das pacientes estudadas, podendo ser considerado elevado, uma vez que a recomendação é a utilização de até uma lata de óleo por mês para uma família de 4 pessoas ${ }^{19}$.

Analisando os dados da Tabela 2, observa-se que o resultado médio encontrado para o Índice de Massa Corporal (IMC) entre as mulheres é considerado elevado, caracterizando excesso de peso. Estes dados são preocupantes, uma vez que o risco de morbimortalidade aumenta com a obesidade ${ }^{3}$. Resultados semelhantes foram encontrados no estudo feito por Gallon, em 2009², onde foram avaliadas 200 pacientes climatéricas entre 40 e 65 anos de idade, e o resultado médio foi de $30,1 \mathrm{Kg} / \mathrm{m}^{2}$. Silva ${ }^{9}$ avaliou 30 mulheres que estavam na menopausa, e em $83,3 \%$ da população apresentou IMC inadequado, com sobrepeso e obesidade.

O sobrepeso e a obesidade são fatores de risco relevantes no desenvolvimento de doenças e agravos não transmissíveis, tais como, doenças do aparelho circulatório, diabetes mellitus, hipertensão arterial sistêmica e cânceres. Estes são responsáveis por produzirem mudanças metabólicas, tais como elevação do colesterol, triglicérides, com consequente aumento da pressão arterial e surgimento da resistência periférica à insulina ${ }^{20}$.

Algumas evidências sugerem que a determinação da circunferência da cintura pode promover de forma prática e sensível correlação entre 
distribuição de gordura e riscos de saúde ${ }^{15}$. No presente estudo, os resultados demonstram que, em média, as mulheres apresentaram risco muito alto para desenvolver doenças cardiovasculares, conforme Tabela 2. Costa et al. ${ }^{21}$, observaram que o valor médio encontrado em $69 \mathrm{mu}-$ lheres de 20 a 59 anos foi de $92,50 \mathrm{~cm}$ de circunferência da cintura, sendo um resultado semelhante ao do presente estudo.

Estudos científicos mostram que a obesidade da parte superior do organismo (gordura visceral), aumenta os riscos para a saúde e é um fator de risco para a mortalidade total, mais que o acúmulo de gordura na parte inferior do organismo (quadris e coxas). Proporções mais elevadas de gordura visceral estão associadas a maiores riscos de resistência à insulina, diabetes, hipertensão e aterosclerose, causando doenças cardiovasculares ${ }^{22}$.

Avaliando o valor médio total do consumo energético, observou-se um consumo inadequado, entretanto não deveria resultar, em média, em um parâmetro antropométrico de excesso de peso (Tabela 3). Isso pode ser resultado de esquecimento, por dificuldade de lembrar exatamente o que ingeriu tanto em quantidade quanto no tipo de alimento consumido, visto que indivíduos com excesso de peso tendem a omitir a quantidade ingerida ${ }^{10}$.

As calorias devem ser ajustadas às necessidades individuais, já que os requisitos de energia diminuem com a idade, principalmente pela queda do metabolismo basal e da atividade física. Embora os valores calóricos variem de acordo com sexo, peso, atividade física e idade, as dietas que ficam abaixo de $1800 \mathrm{Kcal} /$ dia em geral fornecem quantidades inadequadas, principalmente de proteínas e cálcio ${ }^{2}$.

Considerando os valores de referência propostos pela DRI ${ }^{13}$, verificou-se, nesta pesquisa, um consumo adequado tanto de carboidratos, como de proteínas e lipídeos totais, conforme Tabela 3. No estudo feito por Silva, em $2010^{9}$, com $30 \mathrm{mu}-$ lheres que estavam na menopausa, o resultado através do R24h demonstrou que os valores encontrados para cada macronutriente apresentaram-se adequados. Resultados semelhantes também foram encontrados no estudo de Gallon ${ }^{2}$, com 200 pacientes climatéricas entre 40 e 65 anos de idade, e os valores encontrados para carboidrato, proteína e lipídio foram, em média, de $56,9 \%, 16,5 \%$ e $26,5 \%$, respectivamente.

Destaca-se que o consumo adequado de macronutrientes vai favorecer a utilização de carboidrato como fonte de energia, a síntese protei- ca e o fornecimento de ácidos graxos essenciais e vitaminas lipossolúveis, sendo fundamentais na vida humana9.

Como a maioria da população estudada apresentou excesso de peso $(83,3 \%)$, os padrões utilizados para analisar o consumo de gorduras saturada, monoinsaturada, poli-insaturada e colesterol foi o preconizado por Carvalho ${ }^{15}$, sendo $7 \%$ de gordura saturada, $13 \%$ de gordura monoinsaturada, $10 \%$ de gordura poli-insaturada e não mais que $300 \mathrm{mg}$ de colesterol por dia.

Analisando especialmente o consumo lipídico das pacientes climatéricas, observa-se que o consumo de gorduras saturadas esteve acima do preconizado, enquanto que as mono e poli-insaturada estiveram abaixo dos padrões. No entanto, o consumo de colesterol esteve adequado, conforme dados da Tabela 3.

O consumo excessivo de ácidos graxos saturados está amplamente relacionado com elevação dos níveis de colesterol sanguíneo e maior suscetibilidade de doenças cardiovasculares ${ }^{23}$. Do mesmo modo, o colesterol proveniente da dieta, constituinte natural do Reino Animal, também está relacionado com estes tipos de doenças, sendo que o consumo adequado não acarreta prejuízos à saúde de indivíduos saudáveis ${ }^{24}$, o que, em média, pode ser verificado entre as pacientes estudadas (Tabela 3).

As doenças cardiovasculares são a principal causa de morte em mulheres acima de 50 anos. Estudos observacionais revelam que, por volta dos 40 a 50 anos, um número muitas vezes superior de homens do que de mulheres morre por doenças arteriais, principalmente por infarto do miocárdio. Essa relação vai diminuindo rapidamente até que aos 75 e 80 anos, as taxas entre os sexos são muito semelhantes. Dentre os fatores que podem explicar essa perda de proteção contra as doenças cardiovasculares nas mulheres ao redor dos 50 anos, estão as modificações dos hormônios sexuais ${ }^{25}$.

Segundo a Diretriz de prevenção de doença cardiovascular (DCV) em mulheres climatéricas ${ }^{26}$, entre 45 e 64 anos, 1:9 mulheres tem alguma forma de DCV, enquanto esta relação passa a 1:3 após 65 anos de idade. A cada década de vida, a taxa de mortalidade no sexo feminino aumenta de três a cinco vezes. A atividade física regular, a redução de peso, o controle do estresse e os hábitos alimentares mais saudáveis, com redução de sal e colesterol na dieta, são medidas importantes para a redução do risco cardiovascular.

Dados epidemiológicos mostram que populações que vivem no Mediterrâneo possuem me- 
nor risco de desenvolver doenças cardiovasculares, em virtude do tipo de alimentação adotada, em que a principal fonte de gordura é o azeite de oliva (ácido graxo monoinsaturado) ${ }^{27}$.

Os ácidos graxos monoinsaturados, representados pela série ômega-9, o ácido oleico é considerado neutro por não interferir na concentração de colesterol e LDL-C. São encontrados em azeite de oliva, óleo de canola, azeitona, abacate e oleaginosas. Já os ácidos graxos poli-insaturados são representados pela série ômega-6 e ômega-3, cujas fontes alimentares são os óleos vegetais, como o de soja. Estes reduzem o colesterol total e o LDL-C ${ }^{27}$.

Analisando o consumo de cálcio e vitamina $\mathrm{D}$ das mulheres climatéricas, observa-se na Tabela 3 um consumo inadequado conforme o preconizado pela DRI ${ }^{14}$.

No estudo de Nosse et al. ${ }^{1}$, com 31 mulheres climatéricas de 45 a 60 anos, o consumo de cálcio apresentou-se inadequado em 83,87\%, com média de $685,33 \mathrm{mg}$, corroborando com os resultados desta pesquisa, onde o consumo inadequado apresentou-se em $90 \%$ do total de mulheres, com média de 549,63 mg. Resultados semelhantes foram encontrados no estudo de Silva ${ }^{9}$, onde o consumo médio foi de $346,94 \mathrm{mg}$ de cálcio. A alta proporção de ingestão inadequada de cálcio é um dado preocupante, uma vez que pode comprometer a mineralização e a manutenção óssea, contribuindo para o surgimento da osteoporose e aumentando o risco de fraturas ${ }^{5}$.

A osteoporose é considerada pela Organização Mundial da Saúde (OMS) como a "Epidemia Silenciosa do Século", atualmente um problema de saúde pública no mundo inteiro devido ao aumento na expectativa de vida das populações. Afeta indivíduos de maior idade, de ambos os sexos, principalmente mulheres após a menopausa, que também apresentam mais fraturas ${ }^{4}$.

Nesta pesquisa, pôde-se constatar um baixo consumo de cálcio e um excesso de peso em $83,3 \%$ das mulheres estudadas. Esta relação pode estar associada a um consumo inadequado do mineral, pois, de acordo com Leão e Santos ${ }^{28}$, a ingestão de cálcio possivelmente se encontra relacionada ao controle do peso por diversos mecanismos. No trato intestinal, proporciona uma ligação com os ácidos graxos, limitando a sua absorção. A participação do mineral na regulação da temperatura corporal e no aumento da termogênese também tem sido apontada como efeito antiobesidade. Do mesmo modo, Crisóstomo et $\mathrm{al}^{29}$, referem que pacientes submetidos às dietas para perda de peso, com elevados teores de cálcio, mostraram uma redução no balanço corpóreo.

A vitamina D é um hormônio esteroide essencial para a homeostase do cálcio e a manutenção da saúde óssea ${ }^{28}$. Pode ser adquirida por meio da alimentação (carne vermelha, frango, peixes, leite, ovos), medicação e exposição ao sol, no mínimo 15 minutos ao dia, em horários e durante períodos adequados (início da manhã e final da tarde $)^{4}$.

Acredita-se que a insuficiência de vitamina D não seja consequência da menor exposição solar, mas um dos fatores que desencadeia o acúmulo de gordura corporal. Esse processo pode estar ligado ao depósito de vitamina $\mathrm{D}$ nos adipócitos, diminuindo a sua biodisponibilidade e provocando uma cascata de reações pelo hipotálamo que resulta no aumento da sensação de fome e diminuição do gasto energético ${ }^{28}$.

Observando os resultados dos dados bioquímicos encontrados nas mulheres climatéricas (Tabela 4), em média, os triglicerídeos são classificados como ótimos, o colesterol total está limítrofe, sendo que o HDL-C está adequado e o LDL-C, desejável, segundo os padrões de referência ${ }^{17}$.

Os resultados encontrados nesta pesquisa não foram observados no estudo de Tardivo ${ }^{10}$, no qual, entre as pacientes avaliadas, constatou-se que os valores médios plasmáticos de colesterol total e LDL estavam altos, diferentemente do HDL que estava baixo.

As dislipidemias proporcionam modificação no metabolismo dos lipídios com consequentes alterações nas concentrações das lipoproteínas plasmáticas, favorecendo o desenvolvimento das doenças cardiovasculares ${ }^{20}$.

Dentre as morbidades referidas neste estudo, a dislipidemia encontrou-se em destaque em algumas pacientes. Ela pode estar relacionada ao consumo excessivo de gorduras saturadas, como também pôde ser observado. A dislipidemia é um importante fator de risco para o desenvolvimento de doenças coronarianas, com consequente redução da quantidade e qualidade de vida ${ }^{20}$.

\section{Conclusão}

A partir do exposto, foi observado que, em média, as mulheres apresentam excesso de peso com risco muito alto para desenvolver doenças cardiovasculares. Quanto ao consumo alimentar, está adequado em relação aos carboidratos e proteínas. Entretanto, o consumo de gorduras saturada, monoinsaturada e poli-insaturada e dos mi- 
cronutrientes cálcio e vitamina D esteve inadequado, pois não atingiu as recomendações mínimas preconizadas.

Em relação aos dados bioquímicos, em média, estiveram de acordo com os valores ideais estabelecidos, com exceção do colesterol total que esteve limítrofe.
Os resultados demonstram que o cuidado nutricional destas pacientes é importante e deve ser intensificado. Desta forma, poderão ser atingidas as recomendações nutricionais, prevenindo comorbidades específicas desta faixa etária, contribuindo para a qualidade de vida e longevidade das mulheres climatéricas.

\section{Colaboradores}

J Martinazzo trabalhou na pesquisa, coleta de dados e na redação do artigo; GC Ceni, na pesquisa e coleta de dados; GP Zemolin, na análise e discussão de dados, na redação e revisão crítica do artigo; RB Spinelli e VPS Zanardo, na discussão de dados e revisão crítica do artigo.

\section{Agradecimentos}

À URI - Erechim (RS), que através do Ambulatório de Especialidades em Nutrição, possibilitou espaço para a realização desta pesquisa.

\section{Referências}

1. Nosse TM, Moreira SLN, Andrade KC. Avaliação dietética de mulheres climatéricas atendidas em uma clínica-escola de nutrição no município de São Paulo. Rev. Bras Ciências da Saúde 2009; 7(21):26-31.

2. Gallon CW. Perfil nutricional e qualidade de vida de mulheres no climatério [dissertação]. Porto Alegre: Universidade Federal do Rio Grande do Sul; 2009.

3. Montilla RNG, Marucci, MFN, Aldrighi JM. Avaliação do estado nutricional e do consumo alimentar de mulheres no climatério. Rev. Assoc Med Bras 2003; 49(1):91-95.

4. Brasil. Ministério da Saúde (MS). Secretaria de Atenção à Saúde. Departamento de Ações Programáticas Estratégicas. Direitos Sexuais e Direitos Reprodutivos. Manual de atenção à mulher no climatério/ menopausa. Brasília: MS; 2008. [Série A. Caderno no 9. Normas e Manuais Técnicos].

5. Montilla RNG, Aldrighi JM, Marucci MFN. Relação cálcio/proteína da dieta de mulheres no climatério. Rev. Assoc Med Bras 2004; 50(1):52-54.

6. França AP. Estado nutricional e risco de doença cardiovascular de mulheres no climatério atendidas em um ambulatório da cidade de São Paulo [dissertação]. São Paulo: Universidade de São Paulo; 2003.

7. Macedo G. Vitamina D e seus metabólitos. In: Bandeira F, Macedo G, Caldas G, Griz L, Faria M, organizadores. Osteoporose. Rio de Janeiro: Medsi; 2000. p. 329-336.

8. Gallagher ML. Os nutrientes e seu metabolismo. In: Mahan LK, Stump SE, organizadores. Krause, alimentos, nutrição e dietoterapia. 12a Edição. Rio de Janeiro: Elsevier; 2010. p. 39-143.

9. Silva LM. Avaliação Nutricional e Consumo Alimentar de Mulheres na Menopausa [dissertação]. Criciúma: Universidade do Extremo Sul Catarinense; 2010.

10. Tardivo AP. Investigação do consumo alimentar e dos indicadores da composição corporal das mulheres na pós-menopausa [dissertação]. Botucatu: Faculdade de Medicina de Botucatu, UNESP; 2008. 
11. Costa RP, Silva CC. Doenças Cardiovasculares. In: Cuppari L, organizador. Guia de Nutrição: Nutrição Clínica no adulto. 2a Edição. Barueri, São Paulo: Manole; 2005. p. 287-312.

12. Reinstein CS. DietWin Profissional [programa de computador]. Versão 2008 for Windows. Porto Alegre: DietWin; 2008.

13. Institute of Medicine, Food and Nutrition Boarb. Dietary References Intakes. Energy, carbohydrate, fiber, fat, fatty acids, cholesterol, protein, and amino acids. Partes 1 e 2. Washington: National Academy Press; 2002.

14. Institute of Medicine,Food and Nutrition Boarb. Dietary References Intakes for calcium, phosphorus, magnesium, vitamin $D$ and fluoride. Washington: National Academy Press; 1997.

15. Carvalho KMB. Obesidade. In: Cuppari L, organizador. Guia de Nutrição: Nutrição Clínica no adulto. 2a Edição. Barueri: Manole; 2005. p. 149-170.

16. Kamimura MA, Baxmann A, Sampaio LR, Cuppari L. Avaliação Nutricional. In: Cuppari L, organizador. Guia de Nutrição: Nutrição Clínica no adulto. 2a Edição. Barueri: Manole; 2005. p. 89-127.

17. Sociedade Brasileira de Cardiologia. III Diretrizes Brasileiras sobre Dislipidemias e Diretriz de Prevenção da Aterosclerose do Departamento de Aterosclerose da Sociedade Brasileira de Cardiologia. Arq Bras Cardiol 2001; 77(Supl.3):3-8.

18. Brasil. Ministério da Saúde (MS). Secretaria de Atenção à Saúde. Departamento de Atenção Básica. Coordenação-Geral da Política de Alimentação e Nutrição. Guia Alimentar para a População Brasileira: promovendo a alimentação saudável.Brasília: MS; 2005. (Série A. Normas e manuais Técnicos)

19. Santos KMO, Aquino RC. Grupo dos óleos e gorduras. In: Philippi ST, organizador. Pirâmide dos alimentos: Fundamentos básico da nutrição. $1^{\mathrm{a}}$ Edição. Barueri: Manole; 2008. p. 241-292.

20. Lima AN. Fatores Associados ao Excesso de Peso entre os Usuários do Serviço de Promoção à Saúde: Academia da Cidade do Distrito Sanitário Leste de Belo Horizonte - Minas Gerais [dissertação]. Belo Horizonte: Universidade Federal de Minas Gerais; 2009.

21. Costa PRF, Assis AMO, Silva MCM, Santana MLP, Dias JC, Pinheiro SMC, Santos NS. Mudança nos parâmetros antropométricos: a influência de um programa de intervenção nutricional e exercício físico em mulheres adultas. Cad Saude Publica 2009; 25(8):1763-1773
22. Cabrera MAS, Wajngarten M, Gebara OCE, Diament J. Relação do índice de massa corporal, da relação cintura-quadril e da circunferência abdominal com a mortalidade em mulheres idosas: seguimento de 5 anos. Cad Saude Publica 2005; 21(3): 767-775.

23. Lima LP, Sampaio HAC. Caracterização socioeconômica, antropométrica e alimentar de obesos graves. Cien Saude Colet 2007; 12(4):1011-1020.

24. Lottenberg AMP, Buonacorso V. Dislipidemias. In: Cuppari L, organizador. Nutrição nas doenças crônicas não transmissíveis. Barueri: Manole; 2009. p. 191-217.

25. Osório-Wender MC, Accetta SG, Campos LS. Climatério. In: Duncan BB, Schmidt MI, Giugliani ERJ. Medicina ambulatorial: condutas de atenção primária baseadas em evidências. $3^{\text {a }}$ Edição. Porto Alegre: Artmed; 2004. p. 481-491.

26. Sociedade Brasileira de Cardiologia. I Diretriz Brasileira sobre Prevenção de Doenças Cardiovasculares em Mulheres Climatéricas e a Influência da Terapia de Reposição Hormonal (TRH) da Sociedade Brasileira de Cardiologia (SBC) e da Associação Brasileira do Climatério (SOBRAC). Arq Bras Cardiol 2008; 91(Supl.1):1-23.

27. Costa RP, Silva CC, Pimentel IC. Terapia Nutricional nas Doenças Cardiovasculares. In: Silva SMCS, Mura JDAP, organizadores. Tratado de Alimentação, Nutrição e Dietoterapia. 2a Edição. São Paulo: Roca; 2011. p. 773-790.

28. Leão ALM, Santos LC. Consumo de micronutrientes e excesso de peso: existe relação? Rev Bras Epidemiol 2012; 15(1):85-95.

29. Crisóstomo NL, Monte R, Navarro F, Neto JS. Relação da obesidade e cálcio: uma abordagem de estudos realizados. Rev. Brasileira de Obesidade, Nutrição e Emagrecimento 2007; 1(4):16-24.

Artigo apresentado em 06/07/2012

Aprovado em 10/11/2012

Versão final apresentada em 22/11/2012 